\begin{tabular}{|c|l|}
\hline Title & Extracellular matrix metall loproteinase inducer is increased in smokers' bronchoal veolar lavage fluid. \\
\hline Author(s) & Betsuy aku, Tomoko; Tanino, Mishie; Nagai, Katsura; Nasuhara, Y asuy uki; Nishimura, Masaharu; Senior, Robert M. \\
\hline Citation & $\begin{array}{l}\text { A merican Journal of Respiratory and Critical Care Medicine, 168(2), 222-227 } \\
\text { https://doi.org/10.1164/fccm.200301-1030C }\end{array}$ \\
\hline Issue Date & 2003-07-15 \\
\hline Doc URL & http:/hdl.handle.net/2115/17089 \\
\hline Type & article \\
\hline File Information & A JR\&CCM168-2.pdf \\
\hline
\end{tabular}

Instructions for use 


\title{
Extracellular Matrix Metalloproteinase Inducer Is Increased in Smokers' Bronchoalveolar Lavage Fluid
}

\author{
Tomoko Betsuyaku, Mishie Tanino, Katsura Nagai, Yasuyuki Nasuhara, Masaharu Nishimura, \\ and Robert M. Senior
}

First Department of Medicine, Hokkaido University School of Medicine, Sapporo, Japan, and the Departments of Medicine and Cell Biology and Physiology, Washington University School of Medicine, St. Louis, Missouri

\begin{abstract}
Extracellular matrix metalloproteinase inducer (EMMPRIN), also called basigin, is present in the lung during development, but its expression in normal adult lung is minimal. Increases of EMMPRIN have been found in various forms of experimental lung injury. To determine whether EMMPRIN might be involved in alveolar injury/ repair associated with smoking, we developed an ELISA for EMMPRIN and applied it to bronchoalveolar lavage fluids from never-smokers $(n=7)$, former smokers $(n=16)$, and current smokers $(n=58)$. The smoker groups included subjects with emphysema, as determined by high-resolution chest computed tomography. EMMPRIN levels were significantly elevated in current and former smokers $(315 \pm 20$ and $175 \pm 15 \mathrm{pg} / \mathrm{ml} \mathrm{SEM}$, respectively, compared with $31 \pm 7 \mathrm{pg} / \mathrm{ml}$ in never-smokers), but the EMMPRIN levels of smokers with emphysema were not different from smokers without emphysema. Immunohistochemistry of smokers' lung tissue showed EMMPRIN in bronchiolar epithelium and alveolar macrophages, but EMMPRIN mRNA in alveolar macrophages was not different between current and never-smokers. Matrix metalloproteinase- 1 was also detectable in the bronchoalveolar lavage fluid from some smokers but not in never-smokers. These findings indicate that smoking is associated with increased intrapulmonary EMMPRIN. Whether EMMPRIN is involved in smoking-induced lung pathology remains to be determined.
\end{abstract}

Keywords: smoking; emphysema; matrix metalloproteinases; alveolar macrophages

Extracellular matrix metalloproteinase (MMP) inducer (EMMPRIN) is a member of the IgG superfamily (1). EMMPRIN is known by other names: CD147 and M6 in humans; basigin and gp42 in mouse; OX-47 and CE9 in rat; and HT7, neurothelin, and 5A11 in chicken. EMMPRIN is strongly expressed in many tissues during development, including lung (2), but only at low levels in normal adult lung and other tissues (3, 4), suggesting a physiologic role in tissue differentiation and development. EMMPRIN was originally identified on the surface of tumor cells and was termed tumor cell-derived collagenase stimulatory factor (5) because it induced collagenase in adjacent stromal cells $(6,7)$. Its possible role in tumor cell invasion and metastasis is supported by studies showing increased dissemination of tumor cells with high levels of basigin expression (8). Its upregulation in tissues

(Received in original form January 25, 2003; accepted in final form April 22, 2003) Supported by National Heart, Lung, and Blood Institute/National Institutes of Health grants HL 29594 and HL 47328; the Alan A. and Edith L. Wolff Charitable Trust (R.M.S); and the Respiratory Failure Research Group of the Ministry of Health, Labor, and Welfare, Japan, scientific research grant 14,570,532 from the Ministry of Education, Science, Culture, and Sports, Japan (T.B.).

Correspondence and requests for reprints should be addressed to Tomoku Betsuyaku, M.D., Ph.D., First Department of Medicine, Hokkaido University School of Medicine, N-15, W-7, Kita-ku, Sapporo 060-8638, Japan. E-mail: bytomoko@med.hokudai.ac.jp Am J Respir Crit Care Med Vol 168. pp 222-227, 2003 Originally Published in Press as DOI: 10.1164/rccm.200301-1030C on April 24, 2003 Internet address: www.atsjournals.org has been also identified in various nonmalignant conditions, including rheumatoid arthritis $(9,10)$, chronic liver disease (11), heart failure $(12,13)$, ischemic myocardial injury (14), atherosclerosis (15), and experimental ventilator-induced lung injury (16).

MMPs are a family of neutral proteinases that degrade extracellular matrix and modify other substrates (17). MMPs have been implicated in various injury and/or repair processes in the lungs $(18,19)$ and other organs. MMPs are regulated at both pretranscriptional and post-transcriptional levels, and their activities can also be regulated by endogenous inhibitors, including the tissue inhibitors of MMPs, $\alpha_{2}$-macroglobulin, and reversion-inducing cysteine-rich protein with Kazal motifs (RECK) (20). Because several MMPs are increased in various types of human alveolar pathology $(21,22)$ and in experimental bleomycin-induced lung injury (23-25), we recently investigated the expression of basigin (murine EMMPRIN) after bleomycin. We found basigin in fibroinflammatory areas, increased basigin in bronchoalveolar lavage (BAL) fluids, and basigin mRNA upregulated in bronchiolar epithelial cells (26).

Cigarette smoking is a major risk factor for the development of emphysema. Although the molecular mechanisms linking smoking to the pathogenesis of emphysema have not been fully clarified, MMPs have been implicated in its development (27-30). Therefore, we hypothesized that EMMPRIN may be upregulated in smokers' lungs and may be involved in the intrapulmonary expression of MMPs associated with smoking. To examine this possibility, we have developed an ELISA for EMMPRIN and have applied it to BAL fluids from never-smokers and smokers. We have observed that EMMPRIN levels are significantly increased in smokers' BAL fluid compared with never-smokers.

\section{METHODS}

\section{Subjects}

We recruited 81 volunteers (aged 32-80 years), including 58 current smokers, 16 former smokers who had not smoked for at least 1 year, and 7 individuals who had never smoked. The subjects had no respiratory symptoms and denied acute respiratory infections within the preceding 2 months. The serum $\alpha-1$ antitrypsin concentration was more than 160 $\mathrm{mg} / \mathrm{dl}$ in all subjects. Measurements of $\mathrm{VC}, \mathrm{FEV}_{1}$, and diffusing capacity were performed on all subjects (CHESTAC-55V; Chest Co., Tokyo, Japan). High-resolution chest computed tomography scans were performed on all current and former smokers to screen for emphysematous changes, as previously described (31). Three pulmonary physicians who were blinded to any information about the subjects independently evaluated the computed tomography scans for the presence of emphysema. Emphysema was considered present only if all three physicians agreed, regardless of severity. Informed consent was obtained from each subject, and the Ethics Committee of Hokkaido University School of Medicine approved the study.

\section{Sequential BAL and Processing of BAL Fluids}

Current smokers were asked to refrain from smoking for at least 12 hours before the BAL procedure to reduce acute effects of smoking. Four separate $50-\mathrm{ml}$ aliquots of sterile $0.9 \%$ saline were instilled into a segment of the right middle lobe through a wedged flexible fiberoptic 
bronchoscope (Olympus BF-B3R, Tokyo, Japan) and then gently aspirated. The fluid returned from the first $50-\mathrm{ml}$ aliquot was discarded to reduce the contribution from large airways (32). The fluid from the other three aliquots was combined and filtered through several layers of gauze to remove excess mucus and debris, and a sample was taken for cell count and differential. The fluid was then centrifuged at 1,500 rpm for 5 minutes at $4^{\circ} \mathrm{C}$. The cell-free supernatant was aliquoted and stored at $-70^{\circ} \mathrm{C}$. The albumin concentration was measured as previously described (31).

\section{Quantification of EMMPRIN by ELISA}

An enzyme immunoassay was developed for the quantification of EMMPRIN in BAL fluid. The wells of microtiter plates (Immulon \#2; Dynatech Laboratories, Inc., Chantilly, VA) were coated overnight by incubation at room temperature with $1 \mu \mathrm{g} / \mathrm{ml}$ of goat polyclonal antibody raised against recombinant extracellular domain of human EMMPRIN (R\&D Systems, Adbington, UK). After three washes with wash buffer $(0.05 \%$ Tween 20 in phosphate-buffered saline [PBS], pH 7.4), the plate was blocked by PBS containing $1 \%$ bovine serum albumin (BSA), $5 \%$ sucrose, and $0.05 \% \mathrm{NaN}_{3}$ and was incubated at room temperature for a minimum of 1 hour. For the generation of a standard curve, recombinant human EMMPRIN (R\&D Systems) was diluted with $0.1 \%$ BSA and $0.05 \%$ Tween 20 in TBS ( $\mathrm{pH} 7.3$ ) in polypropylene tubes to final concentrations of 1,000, 500, 250, 125, 62.5, 31.3, 15.6, and $7.8 \mathrm{pg} / \mathrm{ml}$. The EMMPRIN used to establish the standard curve showed a single band at approximately $40 \mathrm{kD}$ by Western blotting with the primary antibody, using an enhanced chemiluminescence Western blotting analysis system (Amersham, Aylesbury, UK) as previously described (26) (Figure 1). The plate was then washed three times with wash buffer, and $100 \mu \mathrm{L}$ of unconcentrated BAL fluid or standard were added per well, followed by incubation for 2 hours at room temperature. After three washes with wash buffer, the plate was incubated with $100 \mu \mathrm{L}$ of biotinylated antiextracellular domain of human EMMPRIN antibody (R\&D Systems) at $400 \mathrm{ng} / \mathrm{ml}$ for 2 hours at room temperature. Then the plate was washed three times with wash buffer and $100 \mu \mathrm{L}$ of streptoavidin horseradish peroxidase (R\&D Systems), diluted according to the manufacturer's direction, was added to each well. After 20 minutes, the plate was washed three times and incubated with $100 \mu \mathrm{L}$ of a 1:1 mixture of $\mathrm{H}_{2} \mathrm{O}_{2}$ and tetramethylbenzidine ( $\mathrm{R} \& \mathrm{D}$ Systems) in the dark, and the reaction was stopped with $1 \mathrm{M}$ of sulfuric acid within 30 minutes. The absorbance values were determined at $450 \mathrm{~nm}$ using a microplate reader (Wallac 1,420, Turku, Finland). A linear response was obtained with EMMPRIN standards from 7.8 to $1,000 \mathrm{pg} / \mathrm{ml}$ (Figure 2A). The ELISA had an interbatch coefficient of variation of $8 \%(n=9)$ and an intrabatch coefficient of variation of $14 \%(n=10)$, thus providing precise and sensitive detection of EMMPRIN. All assays were done in duplicate, and the mean values were used. All BAL fluid samples were on the standard curve when measured unconcentrated.

To test the reliability and accuracy of the EMMPRIN ELISA, selected BAL fluids were spiked with 125, 250,500, and 1,000 pg of recombinant EMMPRIN and then assayed for EMMPRIN content. As shown in Figure 2B, assays of spiked BAL fluid consistently reflect the sum of the EMMPRIN in the starting sample plus the EMMPRIN spike.

\section{Immunohistochemistry}

Lung tissue was obtained from nine male smokers at the time of lobectomy for removal of primary lung cancer. These included subjects with and without airflow obstruction by pulmonary function testing. The tissue specimens were fixed in $10 \%$ phosphate-buffered formalin and embedded in paraffin. Five-micrometer sections were deparaffinized in xylene and rehydrated with graded alcohols. Antigenic activity was retrieved by incubation in $10 \mathrm{mM}$ of citrate buffer ( $\mathrm{pH}$ 6.0) in a microwave for 5 minutes. After washing in PBS, the slides were processed for immunohistochemistry using a catalyzed signal amplification (CSA) kit (DAKO Japan, Kyoto, Japan) according to the manufacturer's protocol. The primary antibody was anti-EMMPRIN monoclonal antibody (CHEMICON, Temecula, CA) diluted at 1:500 with PBS.

\section{Quantitative Reverse Transcription-Polymerase Chain Reaction for EMMPRIN}

Alveolar macrophages (AMs) recovered from BAL fluids were adjusted to $1 \times 10^{6}$ per $\mathrm{ml}$ and plated on poly-L-lysine-coated plastic wells

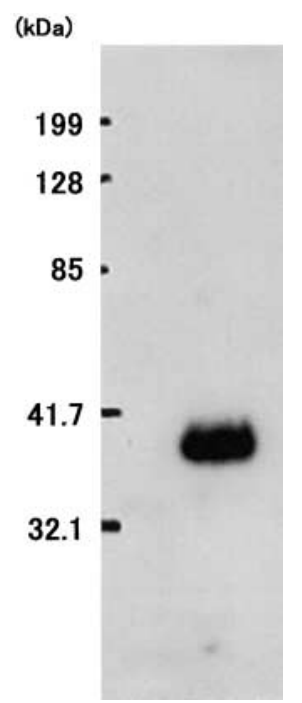

Figure 1. Western blot of recombinant EMMPRIN. The recombinant human EMMPRIN (40 ng) used to establish the standard curve for the EMMPRIN ELISA showed a single band at approximately $40 \mathrm{kDa}$ detected by the goat polyclonal antibody raised against recombinant extracellular domain of human EMMPRIN.

(Corning, NY) for 1 hour at $37^{\circ} \mathrm{C}$ in RPMI-1640 medium (GIBCO, Grand Island, NY). After two washes in PBS, the adherent cells were used for purification of total RNA with guanidium thicyanate using an Isogen RNA extraction kit (Nippon Gene, Tokyo, Japan). Reverse transcription was performed in the presence of Maloney leukemia virus reverse transcriptase (Epicentre Technologies, Madison, WI), and

A
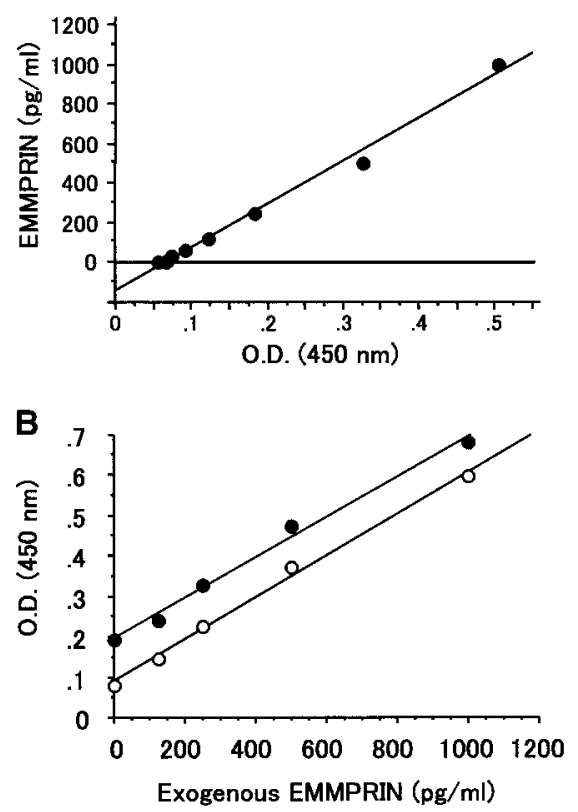

Figure 2. Standard curve of the ELISA for EMMPRIN. (A) A linear response was obtained with concentrations of recombinant EMMPRIN from 7.8 to $1,000 \mathrm{pg} / \mathrm{ml}(r=0.99)$. The concentration of EMMPRIN in BAL fluid was calculated from the standard curve run in parallel with the fluid. $(B)$ One hundred microliters of BAL fluid were spiked with $125,250,500$, and 1,000 pg of exogenous recombinant EMMPRIN, and the samples were assayed for EMMPRIN (closed circles) together with $100 \mu \mathrm{L}$ of $0.1 \% \mathrm{BSA}, 0.05 \%$ Tween 20 in tris-buffered saline $(\mathrm{pH}$ 7.3 ) to which $125,250,500$, and 1,000 pg of exogenous recombinant EMMPRIN was added (open circles). The two sets of samples yielded results that consistently differed by the content of EMMPRIN in the starting BAL fluid. OD = optical density. 
$5^{\prime}$ exonuclease-based fluorogenic polymerase chain reaction was performed using an ABI PRISM 7,700 Sequence Detector (PE Applied Biosystems, Foster City, CA) as described (33). Oligonucleotide primers for EMMPRIN were derived from exon 5 of the human EMMPRIN gene sequence (GenBank accession number: L20471) (34). The primers and probe for this quantification system designed to meet specific criteria by using Primer Express software (PE Applied Biosystems) were as follows: forward, 5'-ACCTGGCCGCCCTCTG -3'; reverse, 5'-TCGTAGTAGAAGATGATGGTGACC-3'; and probe, 5' -FAMCCTTCCATGGCATCGTGGCTGAGGT-TAMRA-3' .

The predicted size of the amplified EMMPRIN DNA product was $83 \mathrm{bp}$, and specific amplification was confirmed by ethidium bromide staining of the polymerase chain reaction product on an agarose gel. Primers and a labeled probe for glyceraldehyde-3-phosphatase-dehydrogenase (GAPDH) cDNA as an endogenous control were purchased from PE Applied Biosystems (TaqMan human GAPDH control reagents). The relative amount of EMMPRIN mRNA in the samples was normalized by GAPDH mRNA.

\section{Interstitial Collagenase (MMP-1)}

MMP-1 levels in BAL fluids were measured by a solid-phase enzyme immmunoassay (MMP-1 Immunoassay Kit; CHEMICON) that recognizes the zymogen and active forms of MMP-1 as well as forms complexed with tissue inhibitor of metalloproteinase-1. Importantly, the assay does not recognize MMP-8, the collagenase expressed by neutrophils. The assay's range of detection was $0.11-3.6 \mathrm{ng} / \mathrm{ml}$. Because MMP-1 was not detected in any unconcentrated BAL fluid, the fluids were concentrated 15 -fold using Centricon filters having a molecular weight cutoff of 10,000 (Amicon Inc., Beverly, MA). Each fluid was measured in duplicate, and the results were averaged.

\section{Statistical Analysis}

Results are expressed as mean \pm SEM unless otherwise stated. Statistical analyses were performed on the data using one-factor analysis of variance among four groups and Student's unpaired $t$ test between two groups when appropriate. The Spearman's rank correlation was calculated to assess the correlation between the data. In all tests, $\mathrm{p}$ values of less than 0.05 were considered statistically significant.

\section{RESULTS}

\section{Characteristics of the Subjects}

The subjects were classified into five groups according to smoking history and presence of emphysema on computed tomography scans (Table 1). There were no significant differences in age among the groups. Emphysema, as detected on computed tomography scans, was less than $25 \%$ of the total lung area in most subjects categorized in the emphysema groups. The $\mathrm{FEV}_{1} / \mathrm{FVC}$ was significantly lower in the current smokers with emphysema than in never-smokers and current smokers without emphysema. Table 2 summarizes findings in the BAL fluids. The concentrations of albumin, cell numbers, and differentials in BAL fluids were not statistically different among the five groups, but the percentage recovery of instilled fluid was significantly lower in current and former smokers with emphysema than in neversmokers $(\mathrm{p}<0.05)$.

TABLE 1. CHARACTERISTICS OF SUBJECTS

\begin{tabular}{|c|c|c|c|c|c|}
\hline & \multirow[b]{3}{*}{ Never-smokers } & \multicolumn{2}{|c|}{ Former Smokers } & \multicolumn{2}{|c|}{ Current Smokers } \\
\hline & & \multicolumn{2}{|c|}{ Emphysema } & \multicolumn{2}{|c|}{ Emphysema } \\
\hline & & $(-)$ & $(+)$ & $(-)$ & $(+)$ \\
\hline Number of subjects & 7 & 9 & 7 & 26 & 32 \\
\hline Age, yr & $64 \pm 3$ & $66 \pm 3$ & $55 \pm 4$ & $53 \pm 2$ & $56 \pm 2$ \\
\hline Pack-year & 0 & $34 \pm 8$ & $50 \pm 10$ & $48 \pm 4$ & $46 \pm 4$ \\
\hline $\mathrm{FEV}_{1} / \mathrm{FVC}, \%$ & $84 \pm 2$ & $79 \pm 2$ & $71 \pm 5$ & $79 \pm 1$ & $71 \pm 2^{\dagger *}$ \\
\hline
\end{tabular}

\footnotetext{
${ }^{\dagger} \mathrm{p}<0.05$ vs. never-smokers.

${ }^{*} p<0.05$ vs. current smokers without emphysema.

Mean \pm SE
}

\section{EMMPRIN in BAL Fluids}

EMMPRIN was detected by ELISA in unconcentrated BAL fluid from all subjects. The EMMPRIN levels were significantly elevated in former and current smokers $(175 \pm 15 \mathrm{pg} / \mathrm{ml}$ SEM and $315 \pm 20$, respectively, compared with $31 \pm 7 \mathrm{pg} / \mathrm{ml}$ in lifelong never-smokers) (Figure 3). However, the levels were not different between the subjects with and without emphysema among former and current smokers. There was a significant but weak correlation between the EMMPRIN level in BAL fluid and the number of AMs in BAL fluid ( $\mathrm{n}=81, \mathrm{p}<0.01, \mathrm{r}=$ 0.43) (Figure 4).

\section{Localization of EMMPRIN in Smokers' Lung}

By immunohistochemistry, EMMPRIN was found predominantly in AMs, bronchiolar epithelium, and bronchial glands in current smokers' lungs. It was present diffusely in the cytoplasm of AMs and bronchial glands (Figures $5 \mathrm{~b}$ and $5 \mathrm{~d}$ ) and was concentrated at the apices of bronchiolar epithelial cells (Figure $5 \mathrm{c}$ ). In contrast, EMMPRIN was weakly seen in AM and was barely detectable in airways in lung tissue from life-long never-smokers (Figure 5a).

\section{EMMPRIN mRNA in AMs}

EMMPRIN mRNA relative to GAPDH mRNA was determined in 21 subjects, including 7 never-smokers and 14 current smokers, including 5 with emphysema. EMMPRIN mRNA/GAPDH mRNA in AM in current smokers with emphysema was increased compared with the others, although it did not reach statistical significance because of the small number of subjects tested (Figure 6). There was a positive but weak relationship between the EMMPRIN concentration in BAL fluid and EMMPRIN mRNA levels in AMs $(\mathrm{n}=21, \mathrm{p}<0.05, \mathrm{r}=0.43)$.

\section{MMP-1}

MMP-1 was not detectable by immunoassay in concentrated BAL fluids from 7 never-smokers but was measurable in concentrated fluids in seven of 36 current smokers, including three with emphysema (Figure 7).

\section{DISCUSSION}

EMMPRIN stimulates the production of MMPs by mesenchymal cells in vitro, including MMP-1, MMP-2, MMP-3, MMP-9, and MMP-14 (6-8, 35). It also induces the expression of MMPs by cells in vivo. For example, human breast cancer cells transfected with EMMPRIN cDNA injected into mammary fat pads of athymic mice have increased expression of MMP-2 and MMP-9 and are more tumorigenic and invasive than control plasmid-transfected cancer cells (8). MMP-1, and possibly other MMPs, binds to EMMPRIN on tumor cells, an effect that may facilitate MMP activity (36). EMMPRIN has been found to interact with cells via a hemo-

\section{TABLE 2. BRONCHOALVEOLAR LAVAGE FLUIDS}

\begin{tabular}{|c|c|c|c|c|c|}
\hline & \multirow[b]{3}{*}{ Never-smokers } & \multicolumn{2}{|c|}{ Former Smokers } & \multicolumn{2}{|c|}{ Current Smokers } \\
\hline & & \multicolumn{2}{|c|}{ Emphysema } & \multicolumn{2}{|c|}{ Emphysema } \\
\hline & & $(-)$ & $(+)$ & & $(+)$ \\
\hline Recovery rate, \% & $71 \pm 4$ & $52 \pm 5$ & $38 \pm 6^{\dagger}$ & $48 \pm 3^{\dagger}$ & $38 \pm 3^{\dagger}$ \\
\hline Albumin, mg/L & $56 \pm 10$ & $51 \pm 8$ & $44 \pm 16$ & $36 \pm 4$ & $47 \pm 6$ \\
\hline Total cells, $\times 10^{-4} / \mathrm{mL}$ & $13 \pm 1$ & $9 \pm 2$ & $10 \pm 2$ & $33 \pm 6$ & $30 \pm 6$ \\
\hline Macrophages, \% & $81 \pm 6$ & $88 \pm 2$ & $81 \pm 7$ & $91 \pm 2$ & $91 \pm 2$ \\
\hline Neutrophils, \% & $0 \pm 0$ & $1 \pm 0$ & $3 \pm 2$ & $2 \pm 1$ & $2 \pm 0$ \\
\hline
\end{tabular}

${ }^{\dagger} \mathrm{p}<0.05$ vs. never-smokers.

Mean \pm SE. 


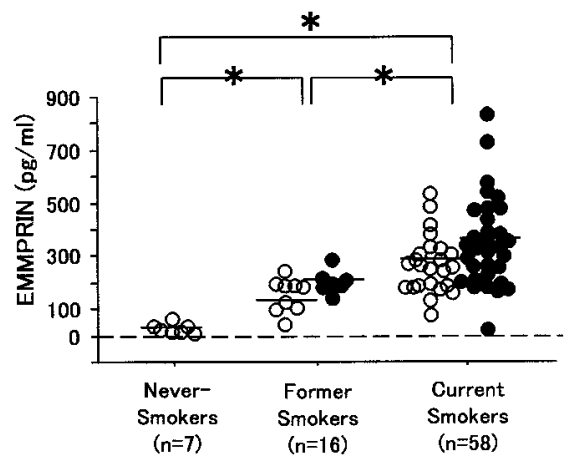

Figure 3. EMMPRIN concentrations in BAL fluids. EMMPRIN levels were significantly elevated in both former and current smokers (179 \pm 17 $\mathrm{pg} / \mathrm{ml} \mathrm{SEM}$ and $319 \pm 20$, respectively, compared with $31 \pm 7$ in never smokers, $p<0.05$ ), but the levels were not significantly different between the subjects with and without emphysema in each group (open circles, without emphysema; closed circles, with emphysema).

philic interaction predominantly through the first immunoglobulin domain (37). Also, EMMPRIN colocalizes with $\alpha 3 \beta 1$ and $\alpha 6 \beta 1$ integrins and can be cross-linked, suggesting an interaction with integrins (38).

Although induction of MMPs is clearly an activity of EMMPRIN, EMMPRIN may have other activities. Complete EMMPRIN deficiency in mice, produced by gene targeting, is associated with a high level of early embryonic lethality (39). Deficient mice that survive have poor growth and abnormalities of the reproductive and central nervous systems (40), and most die before 2 months of age with diffuse infiltration of the lungs with lymphocytes and granulocytes (39).

By Western blotting, EMMPRIN is detectable in BAL fluids from normal rats and mice and is increased in BAL fluids from rats with ventilator-induced lung injury (16) and mice with bleomycin-induced lung fibrosis (26). This study is the first to quantify EMMPRIN in BAL fluid and the first report of EMMPRIN in human BAL fluid. The ELISA that we have developed has enabled reproducible measurements of EMMPRIN in unconcentrated BAL fluid from never-smokers. The average concentration in these subjects is approximately $32 \mathrm{pg} / \mathrm{ml}$. The concentration is approximately 10 -fold higher among current smokers. Former smokers also have elevated levels, approximately fivefold higher than never-smokers despite not smoking for 1 year or longer before obtaining their BAL fluid, indicating that the mechanisms responsible for increased EMMPRIN associated with smoking persist.

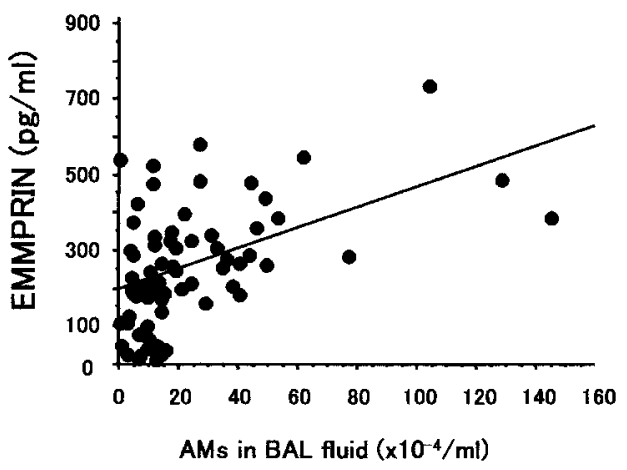

Figure 4. Relationship between AM number in BAL fluid and EMMPRIN concentration. EMMPRIN concentration was weakly correlated to the number of AMs in BAL fluid $\left(\times 10^{-4}\right.$ per $\left.\mathrm{ml}\right)(\mathrm{n}=81, \mathrm{p}<0.01, \mathrm{r}=$ 0.43).
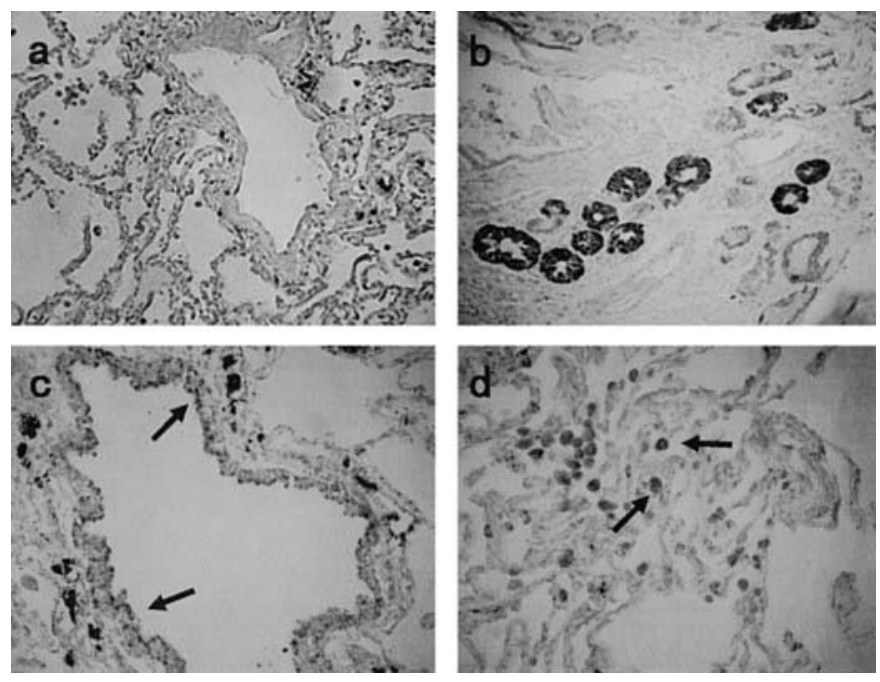

Figure 5. Immunohistochemistry of EMMPRIN in the lungs from neversmokers and smokers. EMMPRIN is weakly present in AM in neversmokers $(a)(\times 200)$. In current smokers with emphysema, EMMPRIN is prominent in bronchial glands $(b)(\times 200)$, bronchiolar epithelium $(c)$ $(\times 400)$, and AMs $(d)(\times 400)$. Arrows indicate bronchiolar epithelium and macrophages, respectively.

The protein core of EMMPRIN is approximately $27 \mathrm{kD}$. Glycosylated forms are 43-66 kD (1), and the capacity to induce MMPs requires glycosylation $(5,37)$. The glycosylation state of EMMPRIN varies among tissues (40). In experimental lung injury, there are increased high molecular weight forms in addition to an increased quantity of EMMPRIN in the BAL fluid $(16,26)$. The EMMPRIN in smokers' BAL fluid in this study also showed multiple molecular weights, corresponding to unglycosylated and glycosylated forms, resembling forms seen in experimentally induced lung injury in rodents and, therefore, might be expected to include EMMPRIN with the capacity to induce MMPs.

The molecular basis for upregulation of EMMPRIN expression in various situations, including the association with smoking, is not known. Likewise, the mechanisms by which EMMPRIN induces MMPs have not been fully established. Binding of Sp1 and $\mathrm{Sp} 3$ to an $\mathrm{Sp} 1$ element in the EMMPRIN promoter appears to be required for regulation of EMMPRIN gene expression in macrophages (41). The levels of Sp1 and Sp3, as well as their activation and binding, are affected by a variety of stimuli so

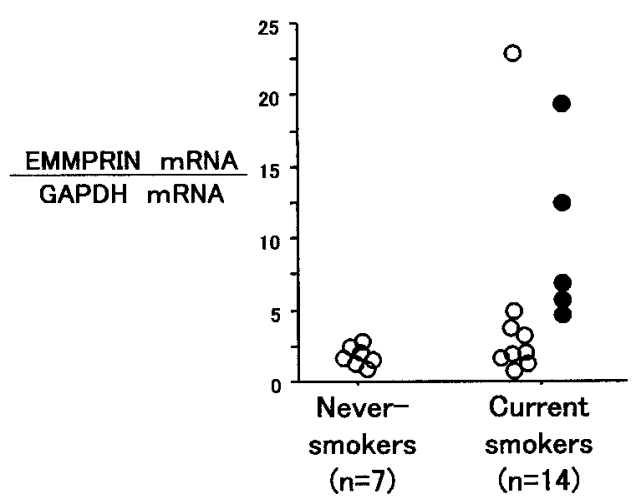

Figure 6. EMMPRIN mRNA in AM. By real-time polymerase chain reaction, EMMPRIN mRNA in AMs was not different between current and never-smokers (open circles, without emphysema; closed circles, with emphysema). 


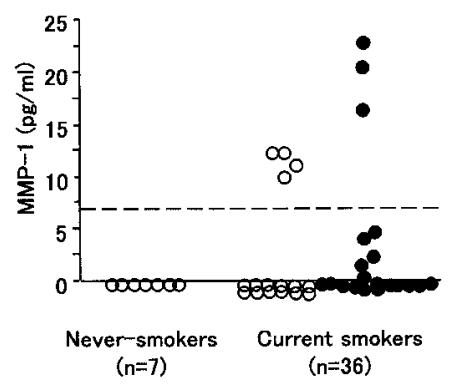

Figure 7. MMP-1 in BAL fluids. MMP-1 was detectable by ELISA in $\times 15$ concentrated BAL fluids from 7 of 36 current smokers, but not in concentrated BAL fluids samples from never-smokers (open circles, without emphysema; closed circles, with emphysema).

that different factors may be involved in different pathologic situations. Intracellular actions of EMMPRIN that lead to MMP induction involve the mitogen-activated protein kinase p38 for MMP-1 (42), but other pathways, including arachidonate metabolism, also appear to be involved (43).

The presence of EMMPRIN in BAL fluid indicates that EMMPRIN can be dissociated from cells despite its presumed transmembrane orientation. Moreover, on Western blot, there is EMMPRIN corresponding to the full-length protein core, suggesting that release of EMMPRIN can occur without proteolytic cleavage. In bleomycin-induced lung injury, we observed that airway and alveolar epithelial cells were EMMPRIN mRNA positive, although EMMPRIN was prominent at fibroinflammatory sites that were essentially devoid of cells expressing EMMPRIN mRNA (26). These findings are consistent with the recent demonstration that breast cancer cells in culture release full-length glycosylated EMMPRIN that promotes release of MMP-2 from fibroblasts $(7,43)$. Other examples of EMMPRIN associated with cells that do not make the protein are peritumor fibroblasts (3). Collectively, the results from these various studies clearly indicate that biologically active EMMPRIN is not restricted to the cells in which it is produced.

Finding increased EMMPRIN in smokers' BAL fluid raises the question of its cellular source. AMs appear to be one likely source, as EMMPRIN mRNA has been reported in AMs in emphysematous lung associated with cancer (44), and EMMPRIN is expressed by monocyte-derived macrophages (15). However, we did not detect differences in EMMPRIN mRNA in AMs between current and never-smokers. The regression analysis of EMMPRIN concentration against AM numbers in BAL fluid or EMMPRIN mRNA/GAPDH mRNA in AM also gave a poor correlation. It is still possible that synthesis of EMMPRIN in $\mathrm{AM}$ in current smokers and/or the increase of AM number in the lung in smokers may contribute to the elevation of EMMPRIN in smokers' BAL fluid. Another possibility is that AM may be only one of several cell types involved in EMMPRIN production in smokers' lungs. Basigin is expressed in epithelial tissues of fetal mouse lungs (2). EMMPRIN has been found immunohistochemically in ciliated cells of normal bronchi and bronchi affected by squamous cell carcinoma (3). Smoking is highly likely in association with these carcinomas. Other cell types also express EMMPRIN, such as fibroblast-like synovial cells (10), but there is no indication that mesenchymal cells are the source of EMMPRIN in smokers' lungs.

MMPs have been implicated in parenchymal lung destruction and repair processes of emphysema and diverse other pulmonary diseases $(18,19)$. Previously, we reported elevated MMP-9 and MMP-8 in BAL fluids from smokers with emphysema compared with smokers without emphysema (29), but these MMPs were likely derived from neutrophils, at least in part, as some of the MMP-9 was complexed with lipocalin, a neutrophil product, and MMP-8 is primarily a neutrophil enzyme (45). However, there are data linking MMP-1, an enzyme that is not produced by neutrophils, to emphysema. D'Armiento and coworkers generated trans- genic mice that expressed human MMP-1 in their lungs, and these animals developed airspace enlargement resembling emphysema (46). Immunochemically, MMP-1 has been localized in AM, bronchial epithelial cells, and alveolar type II cells in emphysema (30, 47 ), and AMs from patients with emphysema produce elevated quantities of MMP-9 and collagenase activity in culture media (27). Therefore, in this study, we focused on the association between MMP-1 and EMMPRIN. Seven current smokers, including three with emphysema, had detectable levels of MMP-1 in 15-fold concentrated BAL fluids, whereas none of the concentrated BAL fluids from never-smokers showed detectable levels of MMP-1, as reported previously (48). It should be noted that emphysema severity was low in the subjects scored as emphysema in this study. However, neither MMP-1 nor EMMPRIN in BAL fluids separated former or current smokers with emphysema from smokers without emphysema. Perhaps the MMP-1 (and EMMPRIN) levels would be higher in individuals with more advanced disease comparable to the individuals studied by Imai and colleagues (30). Although our focus in this study has been directed to the relationship of EMMPRIN to chronic obstructive pulmonary disease, one might speculate that increased EMMPRIN in smokers' lungs may have other effects such as promoting invasion of primary lung tumor cells considering the association between EMMPRIN and invasiveness of other tumor cells.

In summary, we have observed that smoking is associated with increased EMMPRIN in BAL fluid and that the increase persists for a long period after smoking cessation. AMs appear to be one source of the increased EMMPRIN, as EMMPRIN mRNA is increased in some smokers' AMs. Bronchial epithelial cells also show increased EMMPRIN in smokers and may be another source of increased EMMPRIN in BAL fluid. Our findings support the possibility that EMMPRIN has a role in the pathogenesis of emphysema in smokers.

Acknowledgment: The authors thank Ms. Yoko Suzuki for excellent technical assistance and the technical support staff of R\&D Systems for advice in the development of the ELISA for EMMPRIN.

\section{References}

1. Miyauchi T, Kanekura T, Yamaoka A, Ozawa M, Miyazawa S, Muramatsu T. Basigin, a new, broadly distributed member of the immunoglobulin superfamily, has strong homology with both the immunoglobulin V domain and the beta-chain of major histocompatibility complex class II antigen. J Biochem (Tokyo) 1990;107:316-323.

2. Fan QW, Kadomatsu K, Uchimura K, Muramatsu T. Embigin/Basigin subgroup of the immunoglobulin superfamily: different modes of expression during mouse embryogenesis and correlated expression with carbohydrate antigenic markers. Dev Growth Differ 1998;40:277-286.

3. Caudroy S, Polette M, Tournier JM, Burlet H, Toole B, Zucker S, Birembaut P. Expression of the extracellular matrix metalloproteinase inducer (EMMPRIN) and the matrix metalloproteinase-2 in bronchopulmonary and breast lesions. J Histochem Cytochem 1999;47:15751580.

4. Chen X, Kanekura T, Kanzaki T. Expression of Basigin in human fetal, infantile and adult skin and in basal cell carcinoma. J Cutan Pathol 2001;28:184-190.

5. Biswas C, Zhang Y, DeCastro R, Guo H, Nakamura T, Kataoka H, Nabeshima K. The human tumor cell-derived collagenase stimulatory factor (renamed EMMPRIN) is a member of the immunoglobulin superfamily. Cancer Res 1995;55:434-439.

6. Kataoka H, DeCastro R, Zucker S, Biswas C. Tumor cell-derived collagenase-stimulatory factor increases expression of interstitial collagenase, stromelysin, and 72-kDa gelatinase. Cancer Res 1993;53:3154-3158.

7. Guo H, Zucker S, Gordon MK, Toole BP, Biswas C. Stimulation of matrix metalloproteinase production by recombinant extracellular matrix metalloproteinase inducer from transfected Chinese hamster ovary cells. J Biol Chem 1997;272:24-27.

8. Zucker S, Hymowitz M, Rollo EE, Mann R, Conner CE, Cao J, Foda HD, Tompkins DC, Toole BP. Tumorigenic potential of extracellular matrix metalloproteinase inducer. Am J Pathol 2001;158:1921-1928.

9. Konttinen YT, Li TF, Mandelin J, Liljestrom M, Sorsa T, Santavirta S, Virtanen I. Increased expression of extracellular matrix metalloproteinase inducer in rheumatoid synovium. Arthritis Rheum 2000;43: 275-280. 
10. Tomita T, Nakase T, Kaneko M, Shi K, Takahi K, Ochi T, Yoshikawa H. Expression of extracellular matrix metalloproteinase inducer and enhancement of the production of matrix metalloproteinases in rheumatoid arthritis. Arthritis Rheum 2002;46:373-378.

11. Shackel NA, McGuinness PH, Abbott CA, Gorrell MD, McCaughan $\mathrm{GW}$. Insights into the pathobiology of hepatitis $\mathrm{C}$ virus-associated cirrhosis. Am J Pathol 2002;160:641-654.

12. Spinale FG, Coker ML, Heung LJ, Bond BR, Gunasinghe HR, Etoh T, Goldberg AT, Zellner JL, Crumbley AJ. A matrix metalloproteinase induction/activation system exists in the human left ventricular myocardium and is upregulated in heart failure. Circulation 2000;102: 1944-1949.

13. Li YY, McTiernan CF, Feldman AM. Interplay of matrix metalloproteinases, tissue inhibitors of metalloproteinases and their regulators in cardiac matrix remodeling. Cardiovasc Res 2000;46:214-224.

14. Yamani MH, Tuzcu EM, Starling RC, Ratliff NB, Yu Y, Vince DG, Powell K, Cook D, McCarthy P, Young JB. Myocardial ischemic injury after heart transplantation is associated with upregulation of vitronectin receptor (alpha(v)beta3), activation of the matrix metalloproteinase induction system, and subsequent development of coronary vasculopathy. Circulation 2002;105:1955-1961.

15. Major TC, Liang L, Lu X, Rosebury W, Bocan TM. Extracellular matrix metalloproteinase inducer (EMMPRIN) is induced upon monocyte differentiation and is expressed in human atheroma. Arterioscler Thromb Vasc Biol 2002;22:1200-1207.

16. Foda HD, Rollo EE, Drews M, Conner C, Appelt K, Shalinsky DR, Zucker S. Ventilator-induced lung injury upregulates and activates gelatinases and EMMPRIN: attenuation by the synthetic matrix metalloproteinase inhibitor, Prinomastat (AG3340). Am J Respir Cell Mol Biol 2001;25:717-724.

17. Woessener JF Jr. The matrix metalloproteinase family. In: Parks WC, Mecham RP, editors. Matrix metalloproteinases. San Diego: Academic Press; 1998. p. 1-14.

18. Shapiro SD, Senior RM. Matrix metalloproteinases: matrix degradation and more. Am J Respir Cell Mol Biol 1999;20:1100-1102.

19. Parks WC, Shapiro SD. Matrix metalloproteinases in lung biology. Respir Res 2001;2:10-19.

20. Rhee JS, Coussens LM. RECKing MMP function: implications for cancer development. Trends Cell Biol 2002;12:209-211.

21. Fukuda Y, Ishizaki M, Kudoh S, Kitaichi M, Yamanaka N. Localization of matrix metalloproteinases-1, -2 , and -9 and tissue inhibitor of metalloproteinase-2 in interstitial lung diseases. Lab Invest 1998;78: 687-698.

22. Suga M, Iyonaga K, Okamoto T, Gushima Y, Miyakawa H, Akaike T, Ando M. Characteristic elevation of matrix metalloproteinase activity in idiopathic interstitial pneumonias. Am J Respir Crit Care Med 2000; 162:1949-1956.

23. Swiderski RE, Dencoff JE, Floerchinger CS, Shapiro SD, Hunninghake GW. Differential expression of extracellular matrix remodeling genes in a murine model of bleomycin-induced pulmonary fibrosis. Am J Pathol 1998;152:821-828.

24. Bakowska J, Adamson IY. Collagenase and gelatinase activities in bronchoalveolar lavage fluids during bleomycin-induced lung injury. J Pathol 1998;185:319-323.

25. Betsuyaku T, Fukuda Y, Parks WC, Shipley JM, Senior RM. Gelatinase $\mathrm{B}$ is required for alveolar bronchiolization after intratracheal bleomycin. Am J Pathol 2000;157:525-535.

26. Betsuyaku T, Kadomatsu K, Griffin GL, Muramatsu T, Senior RM. Increased basigin in bleomycin-induced lung injury. Am J Respir Cell Mol Biol 2003;28:600-608

27. Finlay GA, O'Driscoll LR, Russell KJ, D'Arcy EM, Masterson JB, FitzGerald MX, O'Connor CM. Matrix metalloproteinase expression and production by alveolar macrophages in emphysema. Am J Respir Crit Care Med 1997;156:240-247.

28. Finlay GA, Russell KJ, McMahon KJ, D'arcy EM, Masterson JB, FitzGerald MX, O'Connor CM. Elevated levels of matrix metalloproteinases in bronchoalveolar lavage fluid of emphysematous patients. Thorax 1997:52:502-506.

29. Betsuyaku T, Nishimura M, Takeyabu K, Tanino M, Venge P, Xu S, Kawakami Y. Neutrophil granule proteins in bronchoalveolar lavage fluid from subjects with subclinical emphysema. Am J Respir Crit Care Med 1999;159:1985-1991.

30. Imai K, Dalal SS, Chen ES, Downey R, Schulman LL, Ginsburg M, D'Armiento J. Human collagenase (matrix metalloproteinase-1) expression in the lungs of patients with emphysema. Am J Respir Crit Care Med 2001;163:786-791.

31. Yoshioka A, Betsuyaku T, Nishimura M, Miyamoto K, Kondo T, Kawakami Y. Excessive neutrophil elastase in bronchoalveolar lavage fluid in subclinical emphysema. Am J Respir Crit Care Med 1995;152: 2127-2132.

32. Rennard SI, Ghafouri M, Thompson AB, Linder J, Vaughan W, Jones K, Ertl RF, Christensen K, Prince A, Stahl MG, et al. Fractional processing of sequential bronchoalveolar lavage to separate bronchial and alveolar samples. Am Rev Respir Dis 1990;141:208-217.

33. Betsuyaku T, Takeyabu K, Tanino M, Nishimura M. Role of secretory leukocyte protease inhibitor in the development of subclinical emphysema. Eur Respir J 2002;19:1051-1057.

34. Guo H, Majmudar G, Jensen TC, Biswas C, Toole BP, Gordon MK. Characterization of the gene for human EMMPRIN, a tumor cell surface inducer of matrix metalloproteinases. Gene 1998;220:99-108.

35. Sameshima T. Nabeshima K, Toole BP, Yokogami K, Okada Y, Goya T, Koono M, Wakisaka S. Glioma cell extracellular matrix metalloproteinase inducer (EMMPRIN) (CD147) stimulates production of membrane-type matrix metalloproteinases and activated gelatinase $\mathrm{A}$ in co-cultures with brain-derived fibroblasts. Cancer Lett 2000;157:177-184.

36. Guo H, Li R, Zucker S, Toole BP. EMMPRIN (CD147), an inducer of matrix metalloproteinase synthesis, also binds interstitial collagenase to the tumor cell surface. Cancer Res 2000;60:888-891.

37. Sun J, Hemler ME. Regulation of MMP-1 and MMP-2 production through CD147/extracellular matrix metalloproteinase inducer interactions. Cancer Res 2001;61:2276-2281.

38. Berditchevski F, Chang S, Bodorova J, Hemler ME. Generation of monoclonal antibodies to integrin-associated proteins: evidence that alpha3beta1 complexes with EMMPRIN/basigin/OX47/M6. J Biol Chem 1997; 272:29174-29180.

39. Igakura T, Kadomatsu K, Kaname T, Muramatsu H, Fan QW, Miyauchi T, Toyama Y, Kuno N, Yuasa S, Takahashi M, et al. A Null mutation in basigin, an immunoglobulin superfamily member, indicates its important roles in peri-implantation development and spermatogenesis. Dev Biol 1998;194:152-165.

40. Fan QW, Yuasa S, Kuno N, Senda T, Kobayashi M, Muramatsu T, Kadomatsu K. Expression of basigin, a member of the immunoglobulin superfamily, in the mouse central nervous system. Neurosci Res 1998; 30:53-63.

41. Liang L, Major T, Bocan T. Characterization of the promoter of human extracellular matrix metalloproteinase inducer (EMMPRIN). Gene 2002. 282:75-86.

42. Lim M, Martinez T, Jablons D, Cameron R, Guo H, Toole B, Li JD, Basbaum C. Tumor-derived EMMPRIN (extracellular matrix metalloproteinase inducer) stimulates collagenase transcription through MAPK p38. FEBS Lett 1998;441:88-92.

43. Taylor PM, Woodfield RJ, Hodgkin MN, Pettitt TR, Martin A, Kerr DJ, Wakelam MJ. Breast cancer cell-derived EMMPRIN stimulates fibroblast MMP2 release through a phospholipase A(2) and 5-lipoxygenase catalyzed pathway. Oncogene 2002;21:5765-5772.

44. Polette M, Gilles C, Marchand V, Lorenzato M, Toole B, Tournier JM, Zucker S, Birembaut P. Tumor collagenase stimulatory factor (TCSF) expression and localization in human lung and breast cancers. $J$ Histochem Cytochem 1997;45:703-709.

45. Borregaard N, Cowland JB. Granules of the human neutrophilic polymorphonuclear leukocyte. Blood 1997;89:3503-3521.

46. D'Armiento J, Dalal SS, Okada Y, Berg RA, Chada K. Collagenase expression in the lungs of transgenic mice causes pulmonary emphysema. Cell 1992;71:955-961.

47. Segura-Valdez L, Pardo A, Gaxiola M, Uhal BD, Becerril C, Selman M. Upregulation of gelatinases $\mathrm{A}$ and $\mathrm{B}$, collagenases 1 and 2, and increased parenchymal cell death in COPD. Chest 2000;117:684-694.

48. Mautino G, Henriquet C, Jaffuel D, Bousquet J, Capony F. Tissue inhibitor of metalloproteinase-1 levels in bronchoalveolar lavage fluid from asthmatic subjects. Am J Respir Crit Care Med 1999;160: 324-330. 